Research Article

\title{
Coherence Properties and Intensity Distribution of a Partially Coherent Lorentz-Gauss Beam Emerging from the Axicon
}

\begin{abstract}
Abdu A. Alkelly (iD and Labiba F. Hassan
Physics Department, Faculty of Science, Sana'a University, Sana'a, Yemen

Correspondence should be addressed to Abdu A. Alkelly; aa_alkelly@yahoo.com

Received 26 September 2021; Accepted 7 December 2021; Published 31 December 2021

Academic Editor: Stefano Trillo

Copyright (C) 2021 Abdu A. Alkelly and Labiba F. Hassan. This is an open access article distributed under the Creative Commons Attribution License, which permits unrestricted use, distribution, and reproduction in any medium, provided the original work is properly cited.

The propagation of a partially Lorentz-Gauss beam in a uniform-intensity diffractive axicon is studied according to the Huygens-Fresnel principle, the Hermite-Gaussian expansion of a Lorentz function, and using the stationary phase method. We have derived the intensity equation of a partially coherent Lorentz-Gauss beams propagating through uniform-intensity diffractive axicon, and we proved mathematically that it is the superposition of Bessel beams of various orders after emerging from axicon, using Hermite's function series and the Bessel function integral formulas. The results show that the intensity distribution of the diffracted beam is the intensity pattern evolved from a Lorentz-Gauss shaped spot into a Gaussian-shaped spot at any position on the focal length of the axicon, and the intensity distribution of a partially Lorentz-Gauss beam generated by an axicon becomes uniform by increasing the beam width and more uniform and constant with the larger coherence width.
\end{abstract}

\section{Introduction}

Recently, the propagation properties of laser beams in axicon have been widely investigated due to their applications such as alignment and metrology, coherence tomography, atom trapping and guiding, optical pumping of plasma, and medical $[1,2]$, so it is considered the most important optical element [3]. The propagation properties of various laser beams through axicon have been illustrated, such as Gaussian beams [4], Laguerre-Gaussian beams [5], Gaussian Schell-model beam [6], and partially coherent flattopped beam [7].

In 2006, Gawhary and Severini [8] introduced a new kind of realizable beam named Lorentz or Lorentz-Gauss beam. These beams are called Lorentz beams because the form of their transverse pattern in the source plane is the product of two independent Lorentz functions. Lorentz-Gauss beams are obtained by multiplying Lorentz beams by Gaussian function. On the other hand, the Lorentz beam has been provided to describe the light field of a diode lasers [9], where the diode lasers have been widely used in practical applications. Since then, the laser beams based on the Lorentz distribution have been widely investigated. The propagation properties of Lorentz and Lorentz-Gauss beams propagating through free space, optical systems, and turbulent atmosphere and oceanic have been widely investigated [10-13], but the propagation properties of a partially coherent Lorentz-Gauss beams through axicon have not been reported. In this study, based on HuygensFresnel integral, we studied the partially coherent Lorentz-Gauss beam generated by annular-aperture logarithmic axicon in Figure 1, and we reported the influences of the beam parameters and coherence properties on the distribution of intensity of Lorentz-Gauss beam by using numerical examples.

\section{Theory}

The electric field of a Lorentz-Gauss beam at $z=0$ is expressed as follows [13]: 


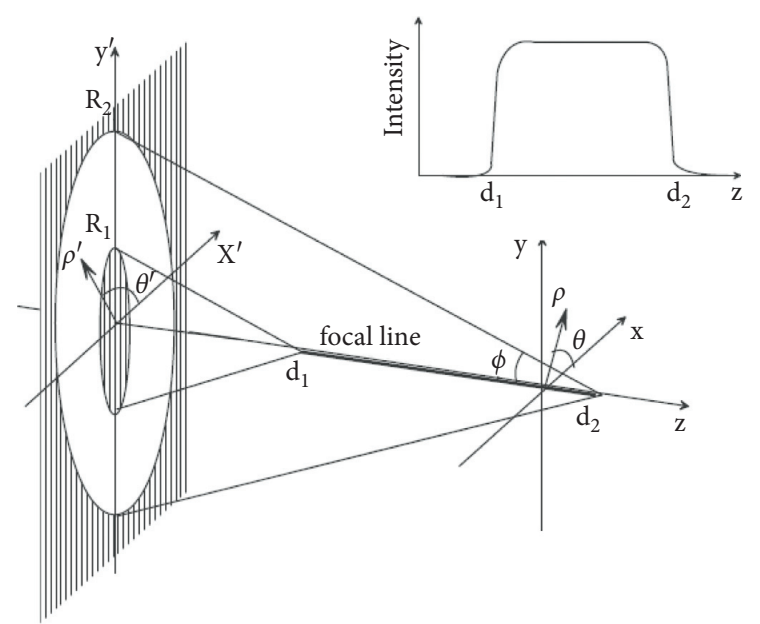

FIGURE 1: Geometry of the annular-aperture diffractive axicon and a notation of the corresponding on-axis profile of image intensity (Figure from [18]).

$$
E\left(\mathbf{r}_{\mathbf{i}}^{\prime}, 0\right)=\frac{1}{w_{0 x} w_{0 y}} \frac{\exp \left(-\mathbf{r}_{\mathbf{i}}^{\prime 2} / w_{0}^{2}\right)}{\left[1+\left(x_{i}^{\prime} / w_{0 x}\right)^{2}\right]\left[1+\left(y_{i}^{\prime} / w_{0 y}\right)^{2}\right]},
$$

where $i=1$ or $2, r^{\prime}=\left(x^{\prime}, y^{\prime}\right)$ is the position vector at the source plane, $z=0$, and $w_{0 x}$ and $w_{0 y}$ are the parameters related to the beam widths of the Lorentz part in $x$ and $y$ direction, respectively. $w_{0}$ is the Gaussian part. Under the condition $w_{0 x}=w_{0 y}=\infty$, equation (1) reduces to the expression for the electric field of Gaussian beam (Figure 2).

The propagation of the partially coherent field means to determine the cross-spectral density function of the field at all pairs of points $r_{1}^{\prime}, r_{2}^{\prime}$ in front side of the surface. The crossspectral density will be expressed as [14]

$$
W\left(\mathbf{r}_{1}^{\prime}, \mathbf{r}_{2}^{\prime}, 0\right)=\left\langle E\left(\mathbf{r}_{1}^{\prime}, 0\right) E^{*}\left(\mathbf{r}_{2}^{\prime}, 0\right)\right\rangle .
$$

where denotes the ensemble average, and * is the complex conjugate.

The cross-spectral density function of a partially coherent Lorentz-Gauss beam generated by a Schell-model source propagating along the $z$ axis at the source plane $z=0$ can be expressed as

$$
\begin{aligned}
W\left(\mathbf{r}_{1}^{\prime}, \mathbf{r}_{2}^{\prime}, 0\right)= & \frac{1}{w_{0 x} w_{0 y}} \frac{\exp \left(-\mathbf{r}_{1}^{\prime 2} / w_{0}^{2}\right)}{\left[1+\left(x_{1}^{\prime} / w_{0 x}\right)^{2}\right]\left[1+\left(y_{1}^{\prime} / w_{0 y}\right)^{2}\right]} \\
& \times \frac{1}{w_{0 x} w_{0 y}} \frac{\exp \left(-\mathbf{r}_{2}^{\prime 2} / w_{0}^{2}\right)}{\left[1+\left(x_{2}^{\prime} / w_{0 x}\right)^{2}\right]\left[1+\left(y_{2}^{\prime} / w_{0 y}\right)^{2}\right]} \\
& \times \exp \left[-\frac{\left(x_{1}^{\prime}-x_{2}^{\prime}\right)^{2}}{2 \sigma_{x}^{2}}-\frac{\left(y_{1}^{\prime}-y_{2}^{\prime}\right)^{2}}{2 \sigma_{y}^{2}}\right],
\end{aligned}
$$

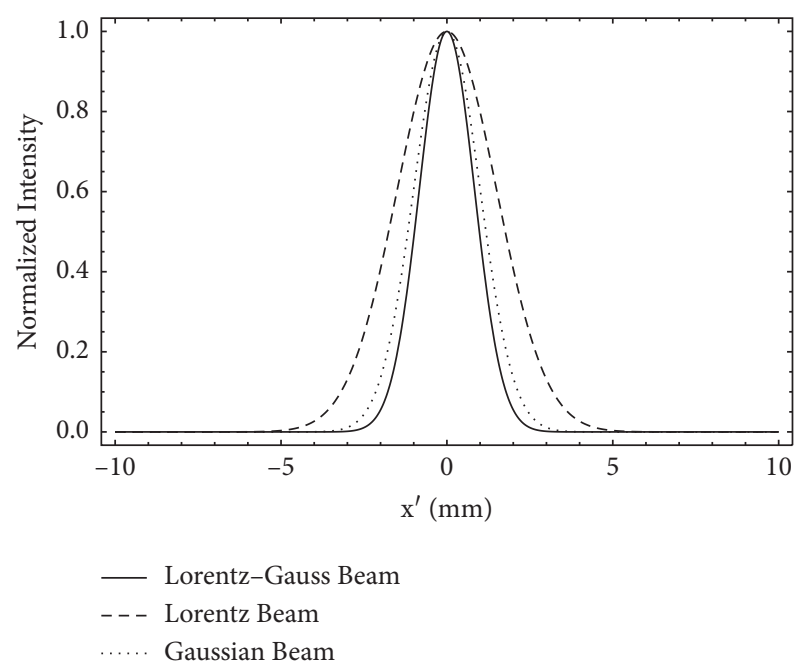

FIgURE 2: Normalized intensity distribution at the source plane along the horizontal direction of Lorentz-Gauss, Lorentz, and Gaussian beam for $w_{0 x}=w_{0 y}=2 \mathrm{~mm}$ and $w_{0}=2 \mathrm{~mm}$.

where $\sigma_{x}$ and $\sigma_{y}$ are the spatial coherence length in the $x$-axis and $y$-axis, respectively. The Lorentz distribution can be expanded as a line superposition of Hermite-Gaussian functions as [15]

$$
\frac{1}{1+\left(x^{\prime} / w_{0 x}\right)^{2}}=\sqrt{\frac{\pi}{2}} \sum_{m=0}^{N} a_{2 m} H_{2 m}\left(\frac{x^{\prime}}{w_{0 x}}\right) \exp \left(-\frac{x^{\prime 2}}{2 w_{0 x}^{2}}\right) \text {, }
$$

where $N$ is the number of expansion, and $a_{2 m}$ is the expanded coefficients that can be expressed as follows [15]:

$$
\begin{aligned}
a_{2 m}= & \frac{(-1)^{m} \sqrt{2}}{(2)^{2 m}}\left\{\frac{1}{m !} \operatorname{erfc}\left(\frac{1}{\sqrt{2}}\right) \exp \left(\frac{1}{2}\right)\right. \\
& +\sum_{b_{1}=1}^{m} \frac{2^{2 b_{1}}}{\left(2 b_{1}\right) !\left(m-b_{1}\right) !}\left[\operatorname{erfc}\left(\frac{1}{\sqrt{2}}\right) \exp \left(\frac{1}{2}\right)\right. \\
& \left.\left.+\sqrt{\frac{2}{\pi}} \sum_{b_{2}=1}^{b_{1}}(-1)^{b_{2}}\left(2 b_{2}-3\right) ! !\right]\right\},
\end{aligned}
$$

where $\operatorname{erfc}($.$) is the complementary error function. With$ increasing the even number $2 m$, the value of $a_{2 m}$ dramatically decreases. Therefore, $N$ will not be large in the calculations; in this work, $N$ is set as $N=1 . H_{2 m}$ is the $2 m$-order Hermite polynomial, and the Hermite polynomial $H_{2 m}(x)$ can be expressed as [16]

$$
H_{2 m}(x)=\sum_{l=0}^{m} \frac{(-1)^{l}(2 m) !}{(l) !(2 m-2 l) !}(2 x)^{2 m-2 l} .
$$

Then, the cross-spectral density function of a partially coherent Lorentz-Gauss beam at the source plane $z=0$ can be rewritten as 


$$
\begin{aligned}
W\left(\mathbf{r}_{1}^{\prime}, \mathbf{r}_{2}^{\prime}, 0\right)= & \frac{\pi^{2}}{4 w_{0 x}^{2} w_{0 y}^{2}} \sum_{m=0}^{N} \sum_{n=0}^{N} \sum_{m^{\prime}=0}^{N} \sum_{n^{\prime}=0}^{N} a_{2 m} a_{2 n} a_{2 m^{\prime}} a_{2 n^{\prime}} \\
& \times H_{2 m}\left(\frac{x_{1}^{\prime}}{w_{0 x}}\right) H_{2 n}\left(\frac{y_{1}^{\prime}}{w_{0 y}}\right) H_{2 m^{\prime}}\left(\frac{x_{2}^{\prime}}{w_{0 x}}\right) H_{2 n^{\prime}}\left(\frac{y_{2}^{\prime}}{w_{0 y}}\right) \\
& \times \exp \left[-\left(\frac{1}{w_{0}^{2}}+\frac{1}{2 w_{0 x}^{2}}\right) x_{1}^{\prime 2}-\left(\frac{1}{w_{0}^{2}}+\frac{1}{2 w_{0 y}^{2}}\right) y_{1}^{\prime 2}\right] \\
& \times \exp \left[-\left(\frac{1}{w_{0}^{2}}+\frac{1}{2 w_{0 x}^{2}}\right) x_{2}^{\prime 2}-\left(\frac{1}{w_{0}^{2}}+\frac{1}{2 w_{0 y}^{2}}\right) y_{2}^{\prime 2}\right] \\
& \times \exp \left[-\frac{\left(x_{1}^{\prime}-x_{2}^{\prime}\right)^{2}}{2 \sigma_{x}^{2}}-\frac{\left(y_{1}^{\prime}-y_{2}^{\prime}\right)^{2}}{2 \sigma_{y}^{2}}\right] .
\end{aligned}
$$

In the cylindrical coordinate, $(x=\rho \cos \theta, y=\rho \sin \theta$, $z=z$ ), equation (7) can be expressed as

$$
\begin{aligned}
W\left(\boldsymbol{\rho}_{1}^{\prime}, \boldsymbol{\rho}_{2}^{\prime}, 0\right)= & \frac{\pi^{2}}{4 w_{0 x}^{2} w_{0 y}^{2}} \sum_{m=0}^{N} \sum_{n=0}^{N} \sum_{m^{\prime}=0}^{N} \sum_{n^{\prime}=0}^{N} a_{2 m} a_{2 n} a_{2 m^{\prime}} a_{2 n^{\prime}} \\
& \times H_{2 m}\left(\frac{\rho_{1}^{\prime} \cos \theta_{1}^{\prime}}{w_{0 x}}\right) H_{2 n}\left(\frac{\rho_{1}^{\prime} \sin \theta_{1}^{\prime}}{w_{0 y}}\right) H_{2 m^{\prime}}\left(\frac{\rho_{2}^{\prime} \cos \theta_{2}^{\prime}}{w_{0 x}}\right) \\
& \times H_{2 n^{\prime}}\left(\frac{\rho_{2}^{\prime} \sin \theta_{2}^{\prime}}{w_{0 y}}\right) \exp \left[-\frac{\rho_{1}^{\prime 2}}{w_{0}^{2}}-\frac{\rho_{2}^{\prime 2}}{w_{0}^{2}}\right] \times \exp \left[-\frac{1}{2}\left(\frac{\rho_{1}^{\prime 2} \cos ^{2} \theta_{1}^{\prime}}{w_{0 x}^{2}}+\frac{\rho_{1}^{\prime 2} \sin ^{2} \theta_{1}^{\prime}}{w_{0 y}^{2}}\right)\right] \\
& \times \exp \left[-\frac{1}{2}\left(\frac{\rho_{2}^{\prime 2} \cos ^{2} \theta_{2}^{\prime}}{w_{0 x}^{2}}+\frac{\rho_{2}^{\prime 2} \sin ^{2} \theta_{2}^{\prime}}{w_{0 y}^{2}}\right)\right] \\
& \times \exp \left[-\left(\frac{\rho_{1}^{\prime 2}+\rho_{2}^{\prime 2}}{2 \sigma^{2}}\right)\right] \exp \left[\frac{\rho_{1}^{\prime} \rho_{2}^{\prime} \cos \left(\theta_{1}^{\prime}-\theta_{2}^{\prime}\right)}{\sigma^{2}}\right] .
\end{aligned}
$$

We considered $\sigma_{x}=\sigma_{y}=\sigma$. The cross-spectral density $W_{\text {out }}\left(\rho_{1}, \rho_{2}\right)$ of the wave field at a pair of points $\rho_{1}=\left(\rho_{1}, \theta_{1}\right)$ and $\rho_{2}=\left(\rho_{2}, \theta_{2}\right)$ behind the axicon $z>0$ can be calculated using the Fresnel diffraction integrals [17]:

$$
\begin{aligned}
W_{\text {out }}\left(\boldsymbol{\rho}_{1}, \boldsymbol{\rho}_{2}, z\right)= & \left(\frac{k}{2 \pi z}\right)^{2} \iint_{A} W_{\text {in }}\left(\boldsymbol{\rho}_{1}^{\prime}, \boldsymbol{\rho}_{2}^{\prime}, 0\right) t\left(\boldsymbol{\rho}_{1}^{\prime}\right) t\left(\boldsymbol{\rho}_{2}^{\prime}\right) \\
& \times \exp \left\{-i k\left[\frac{\left(\boldsymbol{\rho}_{1}-\boldsymbol{\rho}_{1}^{\prime}\right)^{2}-\left(\boldsymbol{\rho}_{2}-\boldsymbol{\rho}_{2}^{\prime}\right)^{2}}{2 z}\right]\right\} \\
& \times \exp \left\{-i k\left[\varphi\left(\boldsymbol{\rho}_{1}^{\prime}\right)-\varphi\left(\boldsymbol{\rho}_{2}^{\prime}\right)\right]\right\} \mathrm{d}^{2} \boldsymbol{\rho}_{1}^{\prime} \mathrm{d}^{2} \boldsymbol{\rho}_{2}^{\prime},
\end{aligned}
$$

where A denotes the axicon aperture.

The optical intensity for the partially coherent light, given by [19], is

$$
I(\boldsymbol{\rho}, z)=W(\boldsymbol{\rho}, \boldsymbol{\rho}, z) .
$$

Because of the rotational symmetry of the transmission function $t\left(\rho^{\prime}\right)$ and the phase $\varphi\left(\rho^{\prime}\right)$ of axicon, i.e., 
$h\left(\rho^{\prime}\right)=t\left(\rho^{\prime}\right) \exp \left[i k \varphi\left(\rho^{\prime}\right)\right]=h\left(\rho^{\prime}\right)$, where $\rho^{\prime}=\left|\rho^{\prime}\right|$, equation (9) after substituting for $W_{\text {in }}\left(\mathbf{r}_{1}^{\prime} ; \mathbf{r}_{2}^{\prime} ; 0\right)$ from equation (8) can be rewritten in form

$$
\begin{aligned}
I_{\text {out }}(\boldsymbol{\rho}, z)= & \frac{k^{2}}{16 z^{2} w_{0 x}^{2} w_{0 y}^{2}} \sum_{m=0}^{N} \sum_{n=0}^{N} \sum_{m^{\prime}=0}^{N} \sum_{n^{\prime}=0}^{N} a_{2 m} a_{2 n} a_{2 m^{\prime}} a_{2 n^{\prime}} \\
& \times \int_{0}^{\infty} \int_{0}^{\infty} t\left(\rho_{1}^{\prime}\right) t\left(\rho_{2}^{\prime}\right) \exp \left[-i k\left(\frac{\rho_{1}^{\prime 2}-\rho_{2}^{\prime 2}}{2 z}\right)\right] \\
& \times \exp \left[-i k\left(\varphi\left(\rho_{1}^{\prime}\right)-\varphi\left(\rho_{2}^{\prime}\right)\right)\right] \exp \left[-\left(\frac{\rho_{1}^{\prime 2}+\rho_{2}^{\prime 2}}{w_{0}^{2}}\right)\right] \\
& \times \exp \left[-\left(\frac{\rho_{1}^{\prime 2}+\rho_{2}^{\prime 2}}{2 \sigma^{2}}\right)\right] G\left(\boldsymbol{\rho}_{1}^{\prime}, \boldsymbol{\rho}_{2}^{\prime}, \boldsymbol{\rho}, z\right) \rho_{1}^{\prime} \rho_{2}^{\prime} \mathrm{d} \rho_{1}^{\prime} \mathrm{d} \rho_{2}^{\prime} .
\end{aligned}
$$

To evaluate the intensity, the integration in equation (11) should can be broken into two parts, one containing the angular integration and the other the radial integration. The angular integration can be performed first, and the angular integration in equation (11) is

$$
\begin{aligned}
G\left(\boldsymbol{\rho}_{1}^{\prime}, \boldsymbol{\rho}_{2}^{\prime}, \rho, z\right)= & \int_{0}^{2 \pi} \int_{0}^{2 \pi} \exp \left[i k \frac{\rho \rho_{1}^{\prime} \cos \left(\theta_{1}-\theta_{1}^{\prime}\right)}{z}\right] \\
& \times \exp \left[-i k \frac{\rho \rho_{2}^{\prime} \cos \left(\theta_{2}-\theta_{2}^{\prime}\right)}{z}\right] \times \exp \left[\frac{\rho_{1}^{\prime} \rho_{2}^{\prime} \cos \left(\theta_{1}^{\prime}-\theta_{2}^{\prime}\right)}{\sigma^{2}}\right] \\
& \times \exp \left\{-\frac{1}{2}\left[\left(\frac{\rho_{1}^{\prime} \cos \theta_{1}^{\prime}}{w_{0 x}}\right)^{2}+\left(\frac{\rho_{1}^{\prime} \sin \theta_{1}^{\prime}}{w_{0 y}}\right)^{2}\right]\right\} \\
& \times \exp \left\{-\frac{1}{2}\left[\left(\frac{\rho_{2}^{\prime} \cos \theta_{2}^{\prime}}{w_{0 x}}\right)^{2}+\left(\frac{\rho_{2}^{\prime} \sin \theta_{2}^{\prime}}{w_{0 y}}\right)^{2}\right]\right\} \\
& \times H_{2 m}\left(\frac{\rho_{1}^{\prime} \cos \theta_{1}^{\prime}}{w_{0 x}}\right) H_{2 n}\left(\frac{\rho_{1}^{\prime} \sin \theta_{1}^{\prime}}{w_{0 y}}\right) \\
& \times H_{2 m^{\prime}}\left(\frac{\rho_{2}^{\prime} \cos \theta_{2}^{\prime}}{w_{0 x}}\right) H_{2 n^{\prime}}\left(\frac{\rho_{2}^{\prime} \sin \theta_{2}^{\prime}}{w_{0 y}}\right) \mathrm{d} \theta_{1}^{\prime} \mathrm{d} \theta_{2}^{\prime} .
\end{aligned}
$$

By using the Jacobian-Anger expansion $e^{i x \cos \theta}=\sum_{m=-\infty}^{\infty} i^{m} J_{m}(x) e^{i m \theta} \quad$ and $\quad e^{x \cos \theta}=\sum_{m=-\infty}^{\infty} I_{m}$ $(x) e^{i m \theta}$, where $J_{m}(x)$ is the Bessel function of the first kind and order $m$, and $I_{p}(x)=i^{-p} J_{p}(i x)$ is the modified Bessel function of the first kind and order $p$, and after integration, the angular integration in equation (12) becomes 
International Journal of Optics

5

$$
\begin{aligned}
G\left(\rho_{1}^{\prime}, \rho_{2}^{\prime}, \rho, z\right)= & 4 \pi^{2} J_{0}\left(k \frac{\rho \rho_{1}^{\prime}}{z}\right) J_{0}\left(k \frac{\rho \rho_{2}^{\prime}}{z}\right) I_{0}\left(\frac{\rho_{1}^{\prime} \rho_{2}^{\prime}}{\sigma^{2}}\right) \\
& \times \exp \left[-\frac{\rho_{1}^{\prime 2}}{4}\left(\frac{1}{w_{0 x}^{2}}+\frac{1}{w_{0 y}^{2}}\right)-\frac{\rho_{2}^{\prime 2}}{4}\left(\frac{1}{w_{0 x}^{2}}+\frac{1}{w_{0 y}^{2}}\right)\right] \\
& \times \sum_{t=0}^{m} \frac{(-1)^{t} 2 m !}{t !}\left(\frac{\rho_{1}^{\prime}}{w_{0 x}}\right)^{2(m-t)} \\
& \times \sum_{s=0}^{n} \frac{(-1)^{s} 2 n !}{s !}\left(\frac{\rho_{1}^{\prime}}{w_{0 y}}\right)^{2(n-s)} T\left(\rho_{1}^{\prime}\right) \\
& \times \sum_{t^{\prime}=0}^{m^{\prime}} \frac{(-1)^{t^{\prime}} 2 m^{\prime} !}{t^{\prime} !}\left(\frac{\rho_{2}^{\prime}}{w_{0 x}}\right)^{2\left(m^{\prime}-t^{\prime}\right)} \\
& \times \sum_{s^{\prime}=0}^{n^{\prime}} \frac{(-1)^{s^{\prime}} 2 n^{\prime} !}{s^{\prime} !}\left(\frac{\rho_{2}^{\prime}}{w_{0 y}}\right)^{2\left(n^{\prime}-s^{\prime}\right)} T\left(\rho_{2}^{\prime}\right),
\end{aligned}
$$

where

$$
\begin{aligned}
& T\left(\rho_{i}^{\prime}\right)=\left\{2 \sum_{k=0}^{(m-t)-1} \frac{1}{[2(m-t)-k] ! k !} \sum_{v=0}^{(n-s)-1} \frac{1}{[2(n-s)-v] ! v !}\right. \\
& \times\left[(-1)^{[(m-t)-k]+2[(n-s)-v]}\right. \\
& \times I_{((m-t)-k)+((n-s)-v)}\left[\frac{\rho_{i}^{\prime 2}}{4}\left(\frac{1}{w_{0 x}^{2}}-\frac{1}{w_{0 y}^{2}}\right)\right] \\
& +(-1)^{[(m-t)-k]} \\
& \left.\times I_{((m-t)-k)-((n-s)-v)}\left[\frac{\rho_{i}^{\prime 2}}{4}\left(\frac{1}{w_{0 x}^{2}}-\frac{1}{w_{0 y}^{2}}\right)\right]\right]+\frac{2}{[(n-s) !]^{2}} \\
& \times \sum_{k=0}^{(m-t)-1} \frac{(-1)^{[(m-t)-k]}}{[2(m-t)-k] ! k !} I_{(m-t)-k}\left[\frac{\rho_{i}^{\prime 2}}{4}\left(\frac{1}{w_{0 x}^{2}}-\frac{1}{w_{0 y}^{2}}\right)\right] \\
& +\frac{2}{[(m-t) !]^{2}} \sum_{v=0}^{(n-s)-1} \frac{(-1)^{2[(n-s)-v]}}{[2(n-s)-v] ! v !} \\
& \times I_{(n-s)-v}\left[\frac{\rho_{i}^{\prime 2}}{4}\left(\frac{1}{w_{0 x}^{2}}-\frac{1}{w_{0 y}^{2}}\right)\right] \\
& \left.+\frac{1}{[(m-t) !]^{2}[(n-s) !]^{2}} I_{0}\left[\frac{\rho_{i}^{\prime 2}}{4}\left(\frac{1}{w_{0 x}^{2}}-\frac{1}{w_{0 y}^{2}}\right)\right]\right\} \text {. }
\end{aligned}
$$


By substituting for $G\left(\rho_{1}^{\prime}, \rho_{2}^{\prime}, \rho, z\right)$ from equations (13)(14) into equation (11), we find the intensity behind the axicon becomes

$$
\begin{aligned}
I_{\text {out }}(\rho, z)= & \frac{k^{2}}{16 z^{2} w_{0 x}^{2} w_{0 y}^{2}} \sum_{m=0}^{N} \sum_{n=0}^{N} \sum_{m^{\prime}=0}^{N} \sum_{n^{\prime}=0}^{N} a_{2 m} a_{2 n} a_{2 m^{\prime}} a_{2 n^{\prime}} \\
& \times \int_{0}^{\infty} \int_{0}^{\infty} t\left(\rho_{1}^{\prime}\right) t\left(\rho_{2}^{\prime}\right) \exp \left[-i k\left(\frac{\rho_{1}^{\prime 2}-\rho_{2}^{\prime 2}}{2 z}\right)\right] \times \exp \left[-i k\left(\varphi\left(\rho_{1}^{\prime}\right)-\varphi\left(\rho_{2}^{\prime}\right)\right)\right] \exp \left[-\left(\frac{\rho_{1}^{\prime 2}+\rho_{2}^{\prime 2}}{w_{0}^{2}}\right)\right] \\
& \times \exp \left[-\left(\frac{\rho_{1}^{\prime 2}+\rho_{2}^{\prime 2}}{2 \sigma^{2}}\right)\right] G\left(\rho_{1}^{\prime}, \rho_{2}^{\prime}, \rho, z\right) \rho_{1}^{\prime} \rho_{2}^{\prime} \mathrm{d} \rho_{1}^{\prime} \mathrm{d} \rho_{2}^{\prime} .
\end{aligned}
$$

The Fresnel diffraction is now expressed as a sum of terms containing radial integration only. By applying the stationary phase method [20] on equation (15), the corresponding intensity behind the axicon at $z$ distance becomes

$$
\begin{aligned}
I_{\text {out }}(\rho, z)= & \frac{k^{2} \rho_{c}^{2} t^{2}\left(\rho_{c}\right)}{16 z^{2} w_{0 x}^{2} w_{0 y}^{2}}\left(\frac{2 \pi}{k \psi^{\prime \prime}\left(\rho_{c}\right)}\right) \exp \left[-\rho_{c}^{2}\left(\frac{2}{w_{0}^{2}}+\frac{1}{\sigma^{2}}\right)\right] \\
& \times \sum_{m=0}^{N} \sum_{n=0}^{N} \sum_{m^{\prime}=0}^{N} \sum_{n^{\prime}=0}^{N} a_{2 m} a_{2 n} a_{2 m^{\prime}} a_{2 n^{\prime}} G\left(\rho_{c}, \rho, z\right),
\end{aligned}
$$

where $\psi\left(\rho_{c}\right)$ and $t\left(\rho_{c}\right)$ differ in accordance with the type of axicon, $\psi\left(\rho_{c}, z\right)=\rho_{c}^{2} / 2 z+\varphi\left(\rho_{c}\right)$ denotes the total phase in $\rho_{c}$, and in the planar coherent illumination, the paraxial phase function of the annular-aperture logarithmic axicon is given by [21]

$$
\varphi\left(\rho^{\prime}\right)=-\frac{1}{2 a} \ln \left[1+\frac{a\left(\rho^{\prime 2}-R_{1}^{2}\right)}{d_{1}}\right],
$$

where $a=\left(d_{2}-d_{1}\right) /\left(R_{2}^{2}-R_{1}^{2}\right)$, and $R_{1}$ and $R_{2}$ are the inner and outer radii of the annular-aperture, respectively. Then, the total phase becomes

$$
\psi\left(\rho_{c}, z\right)=\frac{\rho_{c}^{2}}{2 z}-\frac{1}{2 a} \ln \left[1+\frac{a\left(\rho_{c}^{2}-R_{1}^{2}\right)}{d_{1}}\right] .
$$

To find the stationary point $\rho_{c}$, which particularly governs the value of the integral, this stationary point should satisfy $d \psi\left(\rho_{c}\right) / d \rho_{c}=0$; then, we get

$$
\rho_{c}=\sqrt{\frac{a R_{1}^{2}+z-d_{1}}{a} .}
$$

Within the geometrical optics approach, the phase functions defined by equation (17) should yield a uniform distribution of the on-axis intensity along the focal segment $d_{1} \leq z \leq d_{2}$ in uniform-intensity full coherent illumination, but a rapid oscillation of the axial intensity will occur (specially with relatively coherent light) because of the diffraction from the sharp edges of the aperture. This oscillation could be eliminated by reducing the sharpness of the edges by using an amplitude transmission function (apodization) $t\left(\rho^{\prime}\right)$, that is, unity over most of the annularaperture but falls off smoothly to zero near the edges. One favored amplitude transmission function that is used is the super-Gaussian [22]:

$$
t(\rho)=\exp \left[-\left(\frac{\rho-\bar{r}}{\omega}\right)^{p}\right],
$$

where $\bar{r}=\left(R_{1}+R_{2}\right) / 2$ is the center radius of the annularaperture, $\omega=\left(R_{2}-R_{1}\right) / 2$ represents the width of the apodization, $R_{1}$ and $R_{2}$ are the same as in equation (17), and $p$ is an even integer that controls the softness of the function. Small $p$ gives a soft apodization, whereas large values of $p$ give a hard apodization. Other apodization forms are flattened Gaussian profiles and an arctangent function.

\section{Numerical Results and Discussion}

We will study the properties of a partially coherent Lorentz-Gauss beam after passing through annular-aperture logarithmic axicon using numerical examples. The parameters of the beam and axicon are chosen as follows: wavelength $\lambda=0.8 \mu \mathrm{m}, R_{1}=2.5 \mathrm{~mm}, R_{2}=5 \mathrm{~mm}, d_{1}=100 \mathrm{~mm}$, $d_{2}=200 \mathrm{~mm}$, and $p=14[1]$. 


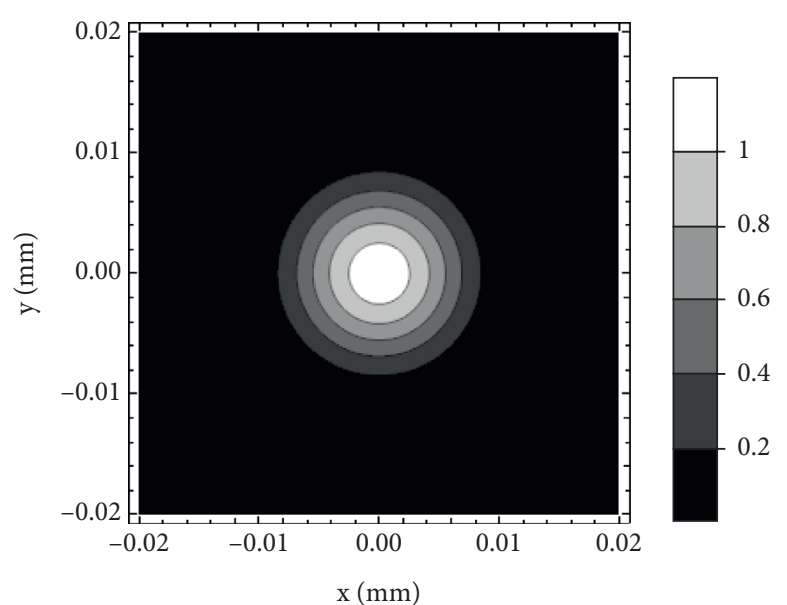

(a)

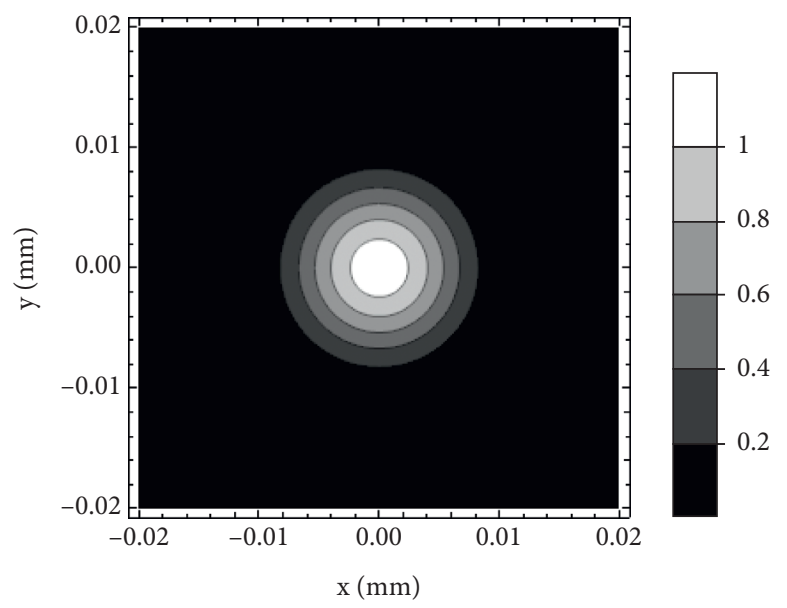

(c)

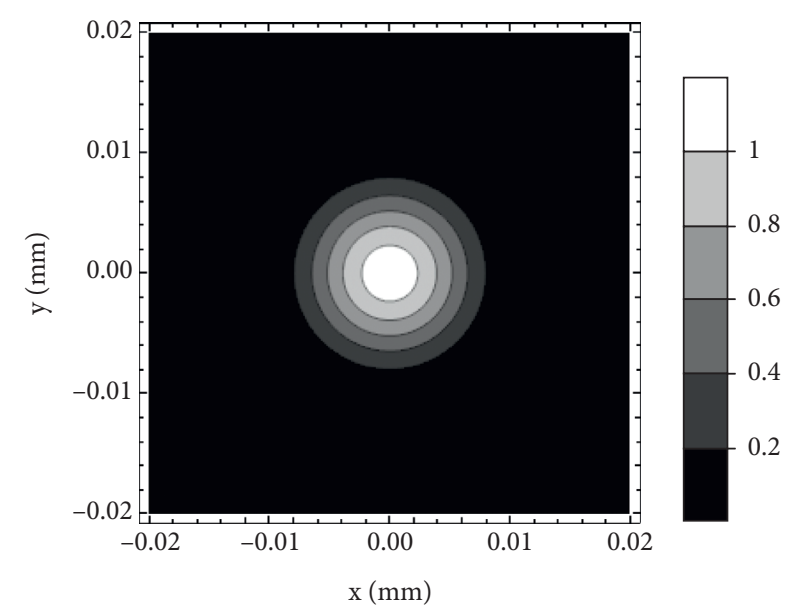

(b)

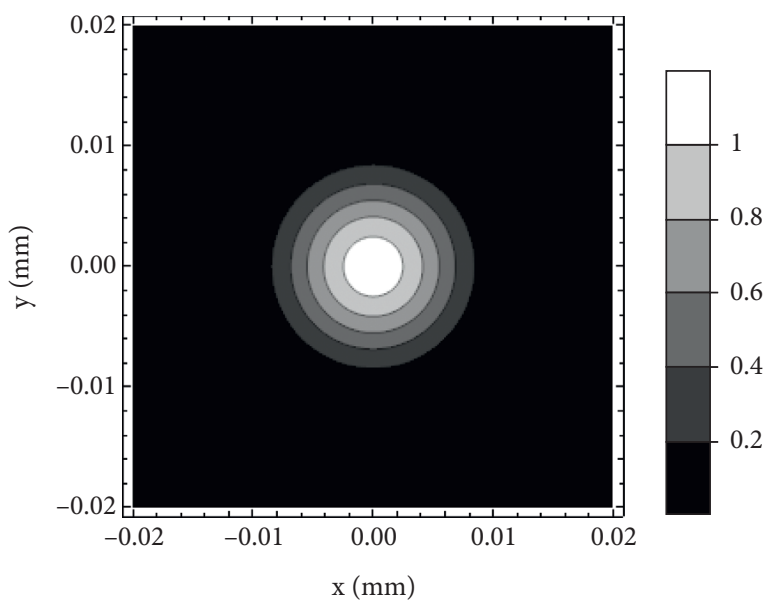

(d)

FIGURE 3: Intensity patterns of the partially coherent Lorentz beam with incident beam widths of $w_{0 x}=2 \mathrm{~mm}, w_{0 y}=5 \mathrm{~mm}, w_{0}=7 \mathrm{~mm}$, with coherence widths $\sigma=8 \mathrm{~mm}$ at different propagation distances in along focal length of logarithmic axicon. (a) $z=100 \mathrm{~mm}$. (b) $z=140 \mathrm{~mm}$. (c) $z=180 \mathrm{~mm}$. (d) $z=200 \mathrm{~mm}$.

Figures 3-6 show the evolution of the intensity pattern of the partially coherent Lorentz-Gauss beam at different propagation distances in along focal length of logarithmic axicon (a) $z=100 \mathrm{~mm}$, (b) $z=140 \mathrm{~mm}$, (c) $z=180 \mathrm{~mm}$, and (d) $z=200 \mathrm{~mm}$. The related width of the Lorentz part has different $w_{0 x}$ and $w_{0 y}$ in Figures 3 and 4 . The waist width $w_{0}$ of Gaussian part is larger than the related width of the Lorentz part of partially coherent Lorentz-Gauss beam in Figure 5, and the waist width $w_{0}$ of the Gaussian part is smaller than the related width of the Lorentz part of partially coherent Lorentz-Gauss beam in Figure 6. From Figures $3-6$, it is illustrated that the partially coherent Lorentz-Gauss beam for different incident beam widths will evolve into a Gaussian-like beam, and the intensities of Figure 3 are seen to be consistent with Figure 4; this indicates that the asymmetry of the incident beam intensity does not affect in the intensity distribution of output Lorentz-Gauss beam behind logarithmic axicon.

For a point lying on the propagation axis $z$, the expression of the intensity distribution is given by substituting $\rho=0$ in equation (16). The computed output axial intensity profiles $I(0, z)$ of a partially coherent Lorentz-Gauss beam for various beam width and various coherence widths $\sigma$ are shown in Figure 7. The case incident beam width of $w_{0 x}=$ $w_{0 y}=3 \mathrm{~mm}, w_{0}=6 \mathrm{~mm}$ is shown in Figure $7(\mathrm{a})$ and displayed the axial intensity profiles $I(0, z)$ for coherence widths $\sigma$ of (a) $0.5 \mathrm{~mm}$, (b) $1 \mathrm{~mm}$, (c) $4 \mathrm{~mm}$, (d) $8 \mathrm{~mm}$, and (e) $\infty$. In each curve, it is seen that the overall intensity drops as the coherence width $\sigma$ decreases, which may be attributed to the energy lost by the reduced coherence of the source, and the curves also have negative slopes within the focal range. Figure 7(b) shows the axial intensity profiles $I(0, z)$ for the same characteristic values of coherence widths $\sigma$ as in Figure $7(\mathrm{a})$, but for Lorentz-Gauss beam intensity distributions with the width of $w_{0 x}=w_{0 y}=15 \mathrm{~mm}, w_{0}=30 \mathrm{~mm}$, we can see in Figure 7(b) that the axial intensity drops as the coherence width decreases, the curves also have negative slopes within the focal range, but the curves increase uniform by increasing the beam width. The curves show, in any beam width state, the on-axis intensities that acquire a 


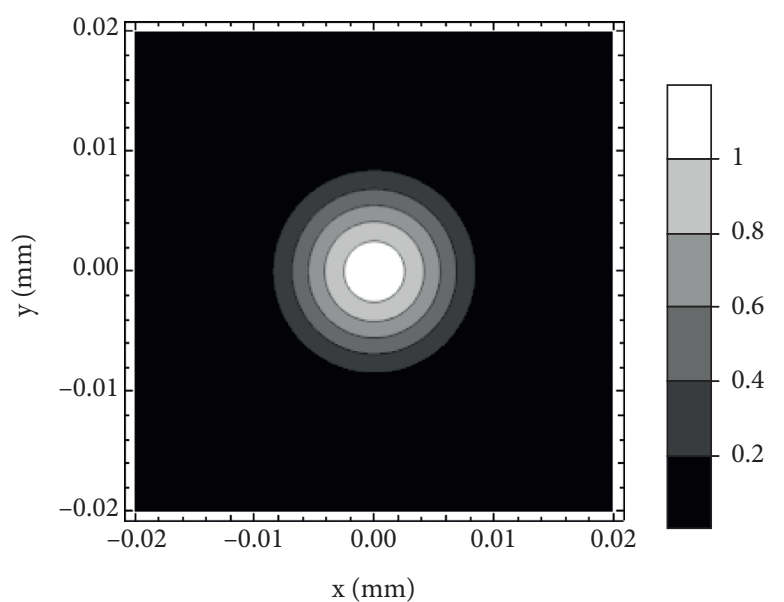

(a)

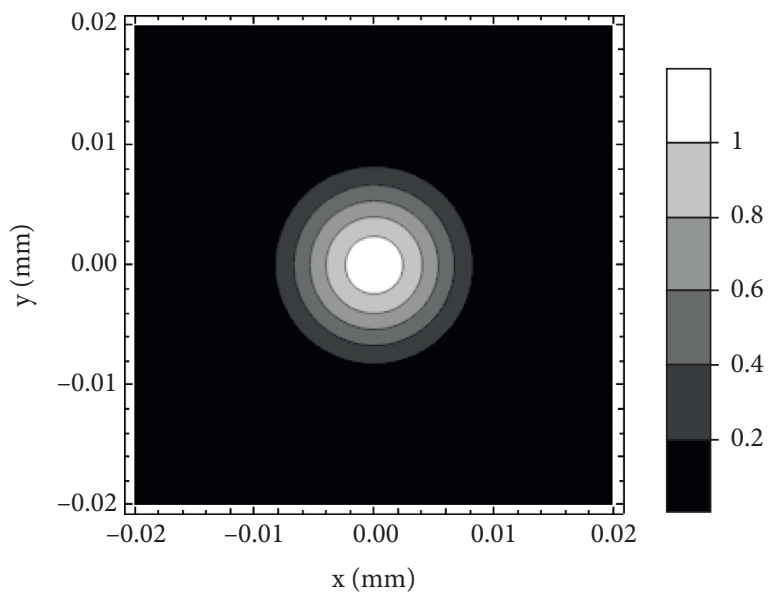

(c)

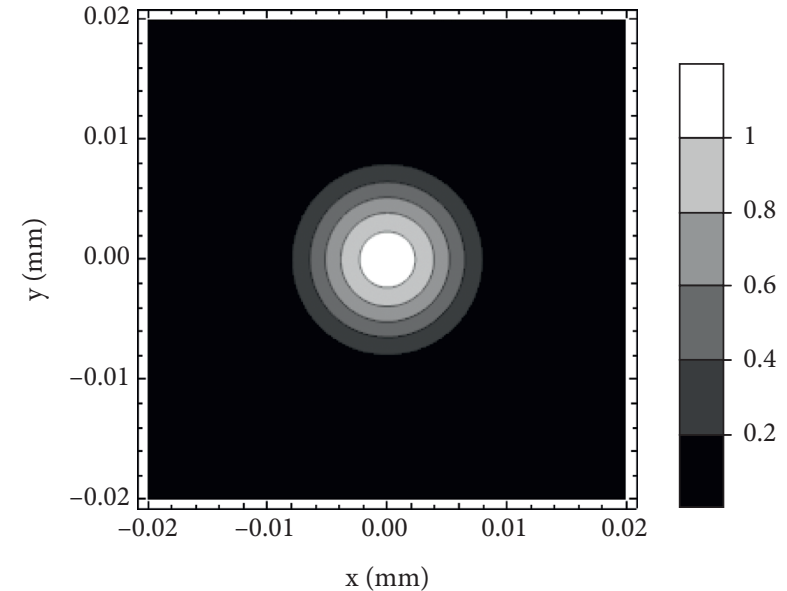

(b)

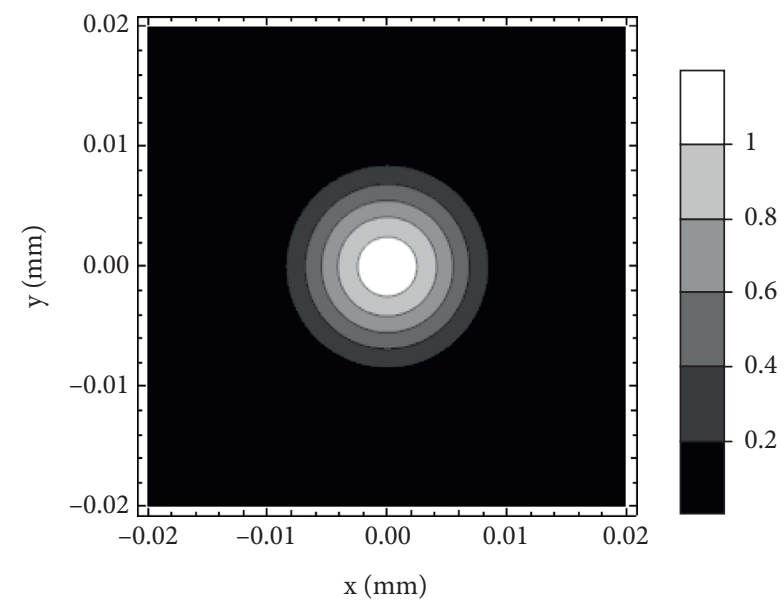

(d)

FIGURE 4: Intensity patterns of the partially coherent Lorentz beam with incident beam widths of $w_{0 x}=5 \mathrm{~mm}, w_{0 y}=2 \mathrm{~mm}, w_{0}=7 \mathrm{~mm}$, with coherence widths $\sigma=8 \mathrm{~mm}$ at different propagation distances in along focal length of logarithmic axicon. (a) $z=100 \mathrm{~mm}$. (b) $z=140 \mathrm{~mm}$. (c) $z=180 \mathrm{~mm}$. (d) $z=200 \mathrm{~mm}$.

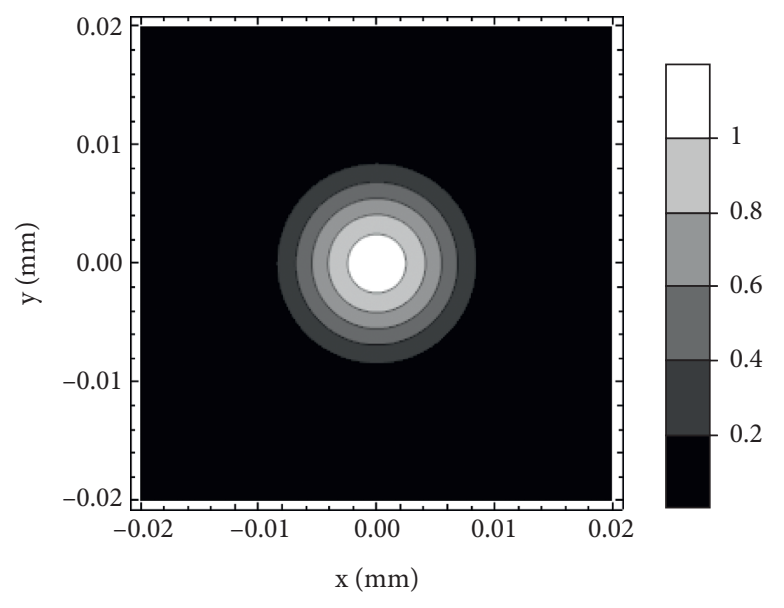

(a)

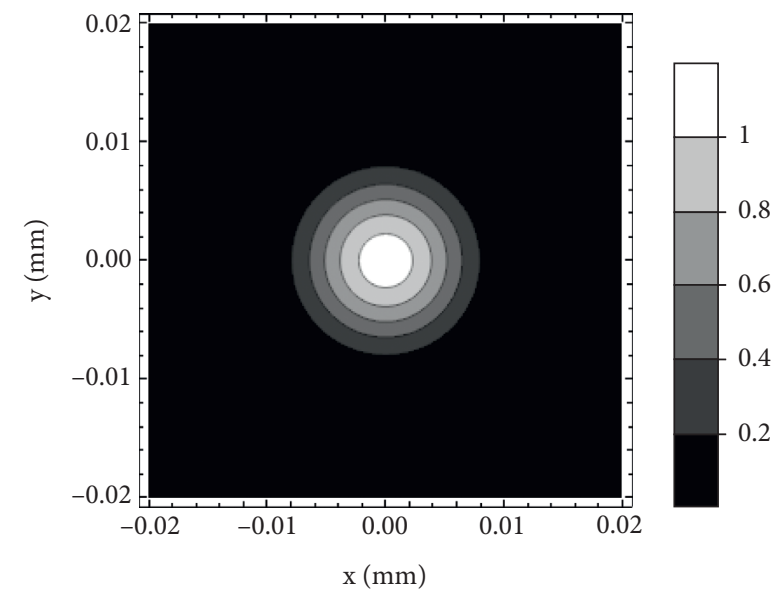

(b)

Figure 5: Continued. 


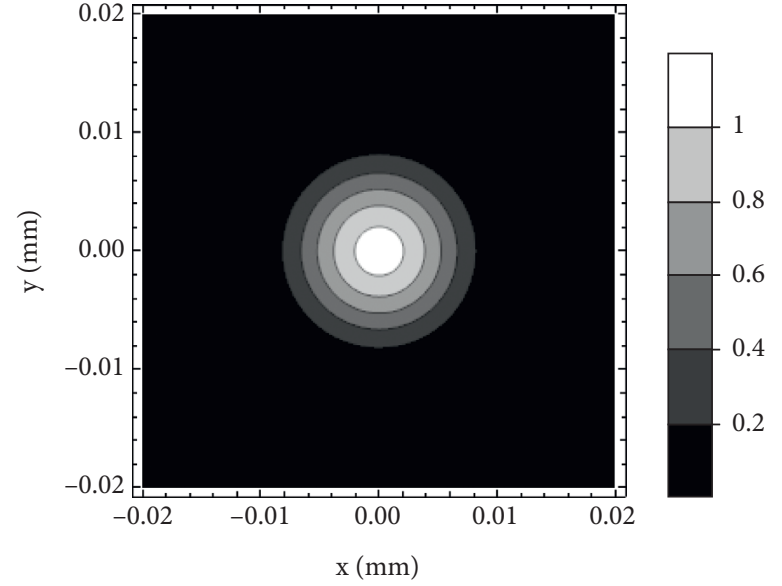

(c)

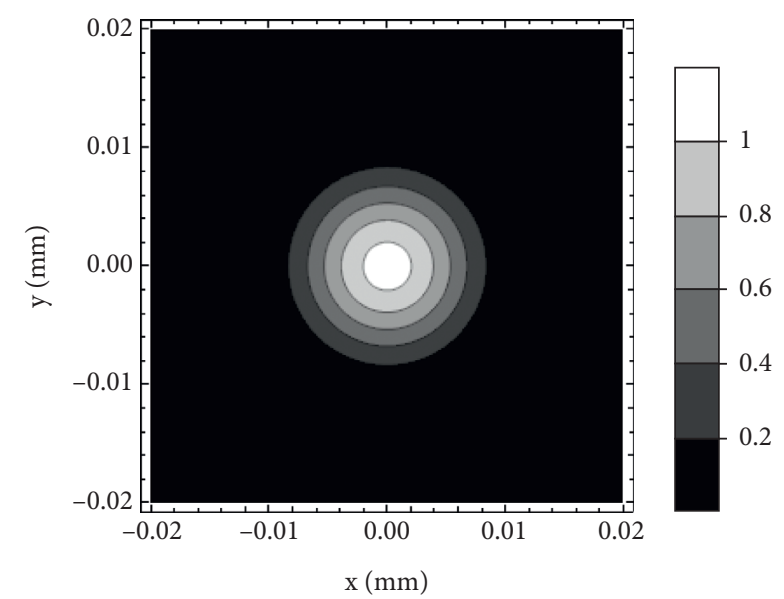

(d)

FIGURE 5: Intensity patterns of the partially coherent Lorentz beam with incident beam widths of $w_{0 x}=w_{0 y}=2 \mathrm{~mm}, w_{0}=7 \mathrm{~mm}$, with coherence widths $\sigma=8 \mathrm{~mm}$ at different propagation distances in along focal length of logarithmic axicon. (a) $z=100 \mathrm{~mm}$. (b) $z=140 \mathrm{~mm}$. (c) $z=180 \mathrm{~mm}$. (d) $z=200 \mathrm{~mm}$.

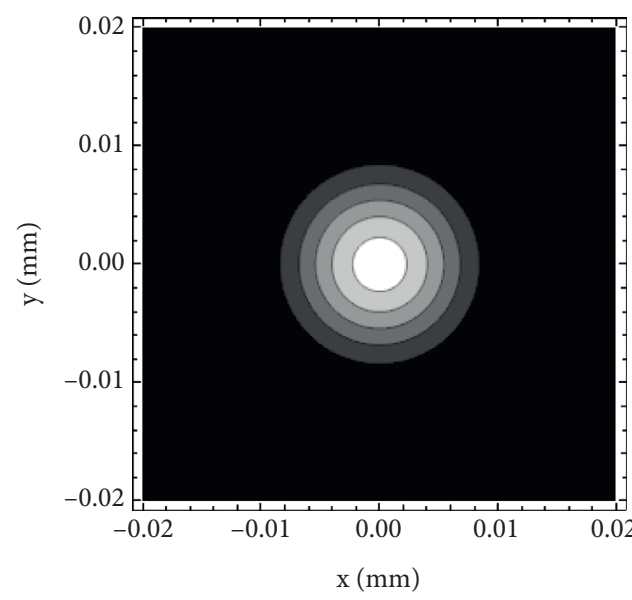

(a)

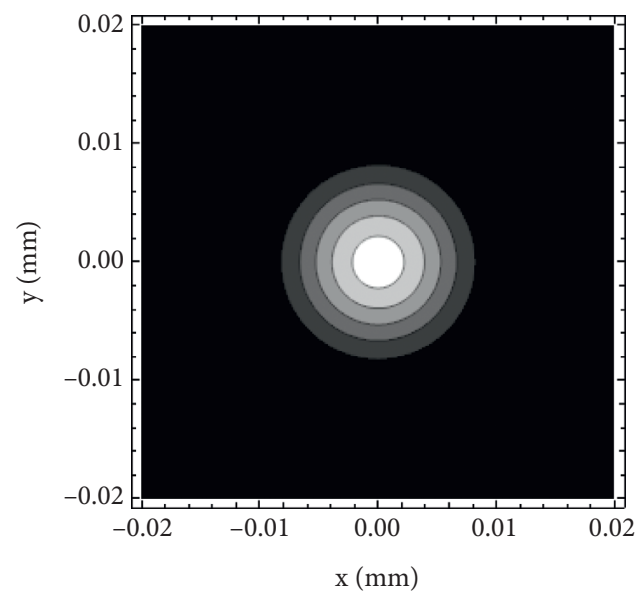

(c)
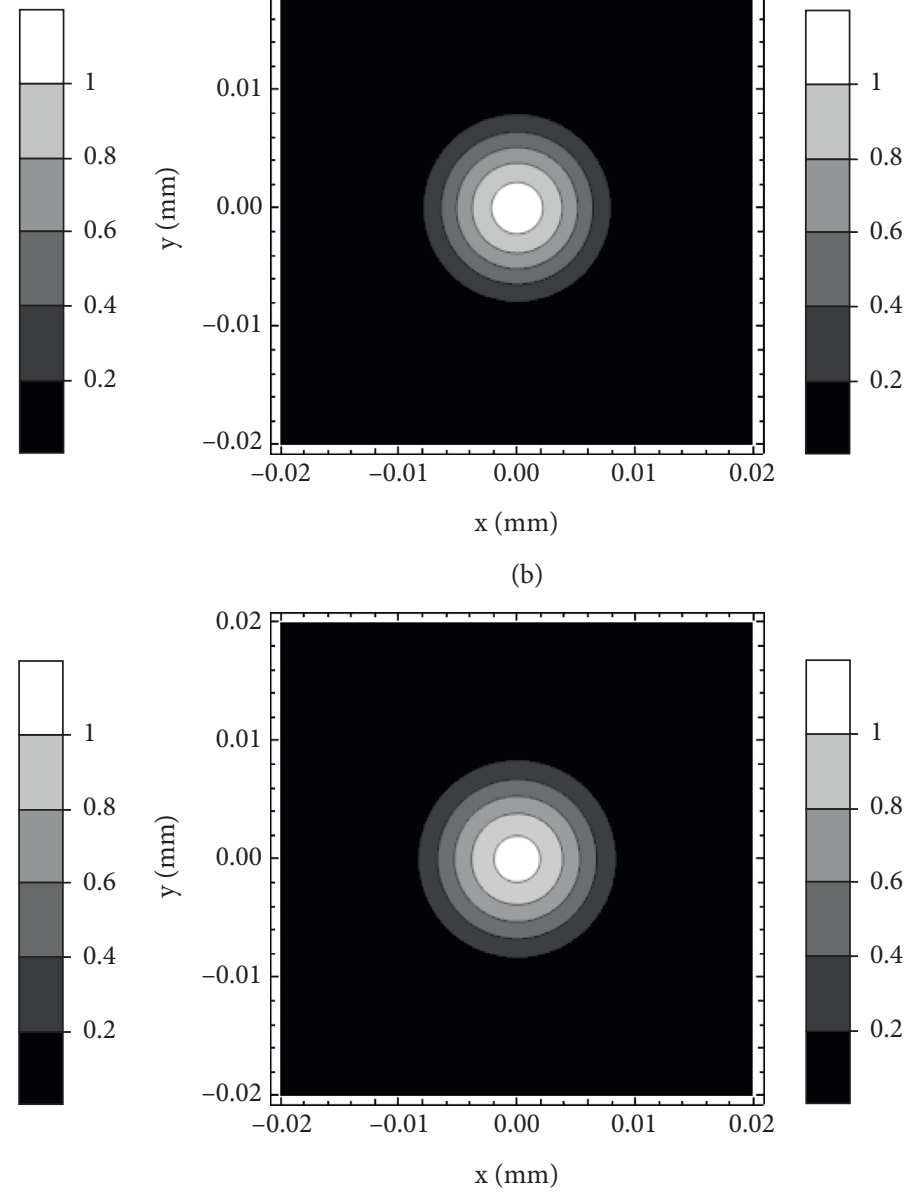

(d)

FIGURE 6: Intensity patterns of the partially coherent Lorentz beam with incident beam widths of $w_{0 x}=w_{0 y}=7 \mathrm{~mm}, w_{0}=2 \mathrm{~mm}$, with coherence widths $\sigma=8 \mathrm{~mm}$ at different propagation distances in along focal length of logarithmic axicon. (a) $z=100 \mathrm{~mm}$. (b) $z=140 \mathrm{~mm}$. (c) $z=180 \mathrm{~mm}$. (d) $z=200 \mathrm{~mm}$. 


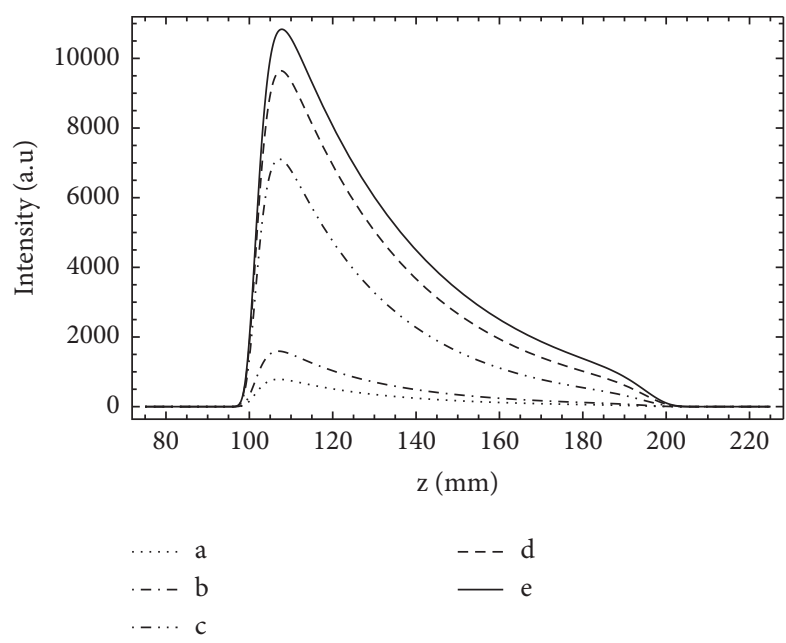

(a)

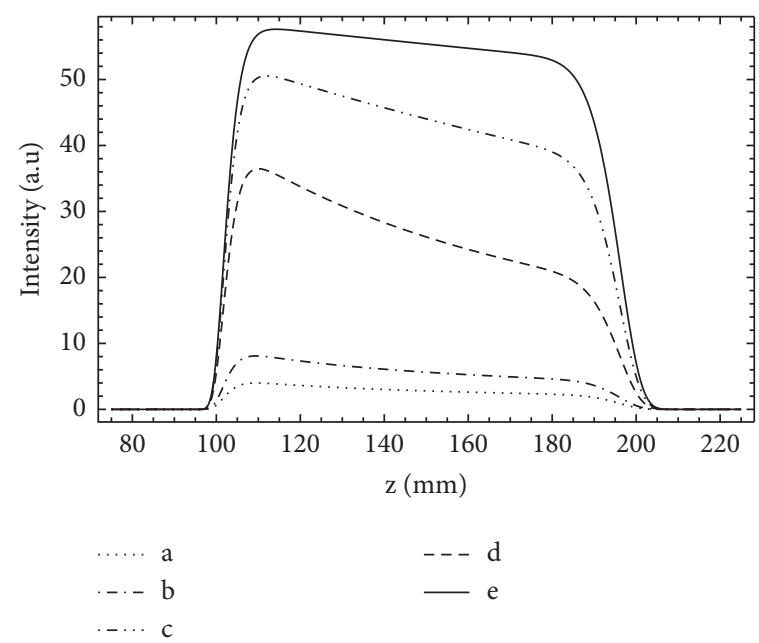

(b)

Figure 7: On-axis intensity distributions I $(0, z)$ produced by an annular-aperture logarithmic axicon illuminated by partially coherent Lorentz-Gauss beam with different incident beam widths of (a) $w_{0 x}=w_{0 y}=3 \mathrm{~mm}, w_{0}=6 \mathrm{~mm}$ and (b) $w_{0 x}=w_{0 y}=15 \mathrm{~mm}, w_{0}=30 \mathrm{~mm}$, with coherence widths $\sigma$ of (A) $0.5 \mathrm{~mm}$, (B) $1 \mathrm{~mm}$, (C) $4 \mathrm{~mm}$, (D) $8 \mathrm{~mm}$, and (E) $\infty$.
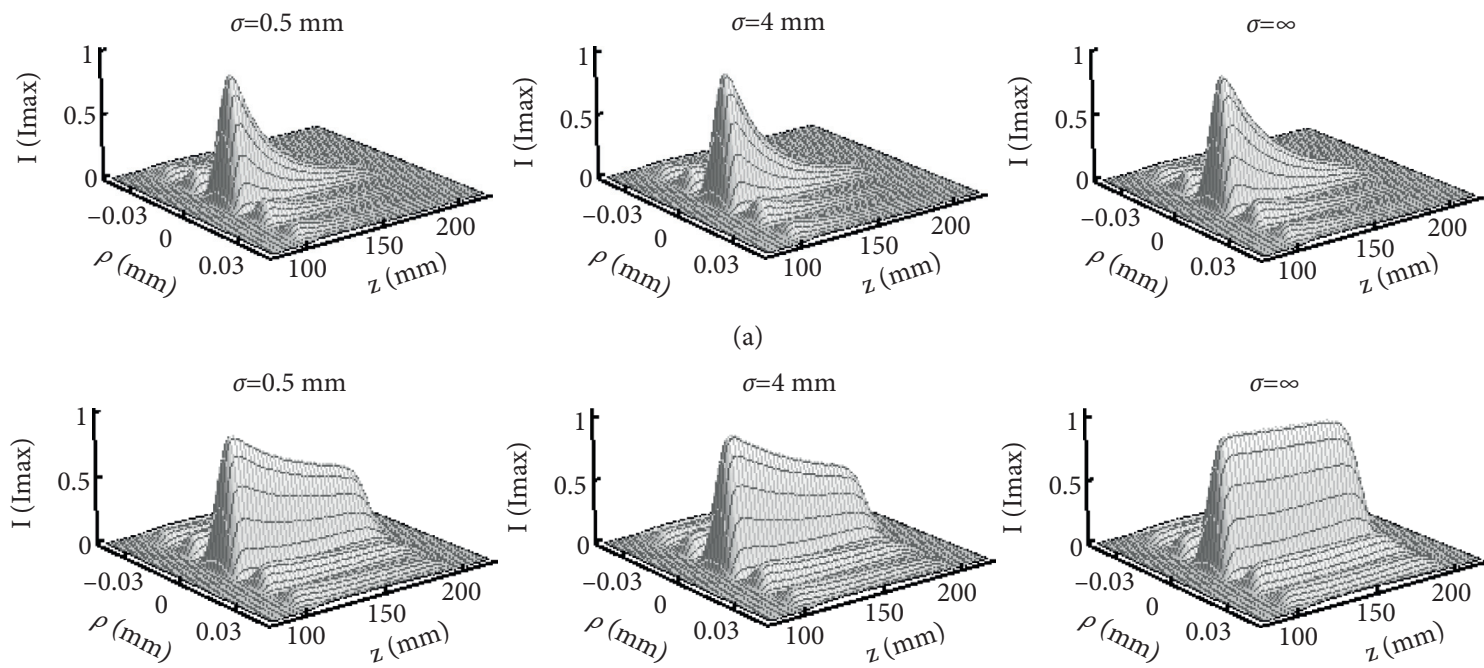

(a)
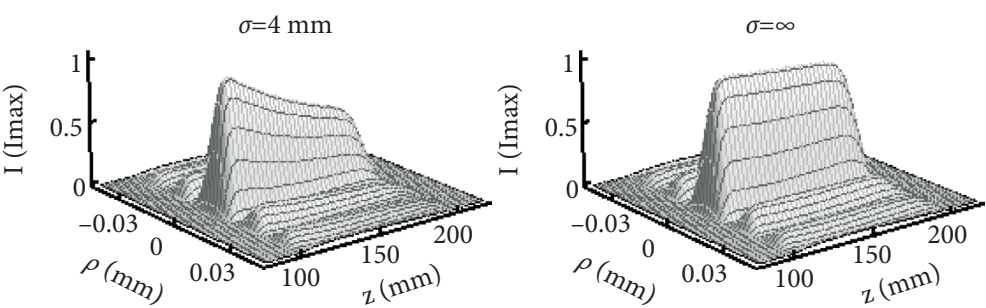

(b)

Figure 8: Normalized intensity distribution produced by annular-aperture logarithmic axicon by partially coherent Lorentz-Gauss beam with incident beam width of (a) $w_{0 x}=w_{0 y}=3 \mathrm{~mm}, w_{0}=6 \mathrm{~mm}$ and (b) $w_{0 x}=w_{0 y}=15 \mathrm{~mm}, w_{0}=30 \mathrm{~mm}$ and different coherence widths.

negative slope whose magnitude decreases as $\sigma$ becomes large, so the effect of coherence is the same for all beam widths. For a larger values of $\sigma$, the axial intensity linearly decreases as a function of distance. The upper curves $\sigma=\infty$ corresponding to a full incident field in Figure 7(b) (E) display the axial intensity that becomes nearly uniform along the focal segment. In each curve, it is seen that when the beam width becomes larger (even with the fully coherent field as in the upper curves (e)), the corresponding axial intensity decreases because the central block of the annularaperture cuts off the increasing rates of the input energy; as a result, more incident energy would be lost, but will produce a uniform beam along the focal segment. We can also note that, for small incident beam width, the curves do not decrease linearly because of the Lorentz-Gauss behavior of the incident distribution.

Figures $8(a)$ and $8(b)$ show the $3 \mathrm{D}$ intensity distributions $I(\rho, z)$ of output Lorentz-Gauss beam behind annular-aperture logarithmic axicon along the focal region $100 \mathrm{~mm} \leq z \leq 200 \mathrm{~mm}$, for several typical values of beam width and coherence width $\sigma$. The slices intensities of Figures $8(\mathrm{a})$ and $8(\mathrm{~b})$ are seen to be consistent with the corresponding curves in Figure 7. Figures 8(a) and 8(b) show the $3 D$ intensity profiles $I(\rho, z)$ for beam widths $w_{0 x}=$ $w_{0 y}=3 \mathrm{~mm}, w_{0}=6 \mathrm{~mm}$ and $w_{0 x}=w_{0 y}=15 \mathrm{~mm}, w_{0}=$ $30 \mathrm{~mm}$, respectively, and coherence widths $\sigma$ of $0.5 \mathrm{~mm}$, 
$4 \mathrm{~mm}$, and $\infty$, and one can see that in several values of beam width, the focal line is narrow with uniform width, approximately $0.02 \mathrm{~mm}$, along the focal segment, except the decay at the far distances in Figure 8(a). By comparing the scale of Figure 8 , one can see that, in fully coherent illumination $\sigma=\infty$ displayed in Figure 8(b), the image profiles show that the intensity becomes more uniform along the line segment and constant along the focal segment.

\section{Conclusions}

In this study, we derived the intensity equation of the partially Lorentz-Gauss beam propagating through the axicon, and we proved mathematically that it is the superposition of Bessel beams of various orders after emerging from axicon by finding the angular integral in equations (13)-(14), using Hermite's function series and the Bessel function integrals formulas. The partially coherent Lorentz-Gauss beam for different incident beam widths will evolve into a Gaussian-like beam, and the asymmetry of the incident beam intensity does not affect in the intensity distribution of the output Lorentz-Gauss beam behind the logarithmic axicon. The intensity distributions have been illustrated and analyzed by using numerical examples. The results show that the intensity distribution of the diffracted beam becomes uniform by increasing the beam width, for larger coherent width will be uniform line and constant along the focal segment.

\section{Data Availability}

The (angular integration in equation (11)) data used to support the findings of this study are included within the supplementary information file (appendix A). The stationary phase method, that we applied it to equation (15), the total phase, and transmission function of the annular-aperture logarithmic axicon studies are cited at relevant places within the text as references [19-21].

\section{Conflicts of Interest}

The authors declare that they have no conflicts of interest.

\section{References}

[1] A. Burvall, K. Kołacz, Z. Jaroszewicz, and A. T. Friberg, "Simple lens axicon," Applied Optics, vol. 43, pp. 4838-4844, 2004.

[2] S. Castillo, "Axicons in action," Optik \& Photonik, vol. 11, no. 5, p. 12, 2017.

[3] Z. Jaroszewicz, A. Burvall, and A. T. Friberg, "Axicon-the most important optical element," Optics and Photonics News, vol. 16, no. 4, pp. 34-39, 2005.

[4] J. Pu, H. Zhang, S. Nemoto, W. Zhang, and W. Zhang, "Annular-aperture diffractive axicons illuminated by gaussian beams," Journal of Optics A: Pure and Applied Optics, vol. 1, no. 6, pp. 730-734, 1999.

[5] V. Jarutis, R. Paškauskas, and A. Stabinis, "Focusing of laguerre-gaussian beams by axicon," Optics Communications, vol. 184, no. 1-4, pp. 105-112, 2000.
[6] A. A. Alkelly, M. Shukri, and Y. S. Alarify, "Intensity distribution and focal depth of axicon illuminated by gaussian schell-model beam," Optics Communications, vol. 284, no. 19, pp. 4658-4662, 2011.

[7] F. M. Thabit, A. A. AlKelly, and M. A. Shukri, "Propagation of fully and partially coherent flat-topped multi-Gaussian beams through axicons," Journal of the Optical Society of America A, vol. 37, no. 5, pp. 759-767, 2020.

[8] O. E. Gawhary and S. Severini, "Lorentz beams and symmetry properties in paraxial optics," Journal of Optics A: Pure and Applied Optics, vol. 8, no. 5, pp. 409-414, 2006.

[9] J. Yang, T. Chen, G. Ding, and X. Yuan, "Focusing of diode laser beams: a partially coherent lorentz model," Semiconductor Lasers and Applications, vol. III, p. 6824, 2008.

[10] G. Zhou, "Propagation of a partially coherent lorentz-gauss beam through a paraxial ABCD optical system," Optics Express, vol. 18, no. 5, pp. 4637-4643, 2010.

[11] G. Zhou and X. Chu, "Average intensity and spreading of a lorentz-gauss beam in turbulent atmosphere," Optics Express, vol. 18, no. 2, pp. 726-731, 2010.

[12] D. Liu, H. Yin, G. Wang, and Y. Wang, "Propagation properties of a partially coherent lorentz beam in uniaxial crystal orthogonal to the optical axis," Journal of the Optical Society of America A, vol. 34, no. 6, pp. 953-960, 2017.

[13] D. Liu, G. Wang, and Y. Wang, "Average intensity and coherence properties of a partially coherent lorentz-gauss beam propagating through oceanic turbulence," Optics \& Laser Technology, vol. 98, pp. 309-317, 2018.

[14] L. Mandel and E. Wolf, "Optical Coherence and Quantum Optics", Optical Coherence and Quantum Optics, Cambridge University Press, Cambridge, UK, 1995.

[15] P. P. Schmidt, "A method for the convolution of lineshapes which involve the lorentz distribution," Journal of Physics B: Atomic and Molecular Physics, vol. 9, no. 13, pp. 2331-2339, 1976.

[16] H. H. D. Alan Jeffrey, Handbook of Mathematical Formulas and Integrals, Academic Press, Cambridge, MA, USA, 4th edition, 2008.

[17] T. Ari, "Friberg, "Stationary-phase analysis of generalized axicons," Journal of Optical Society America A, vol. 13, pp. 743-750, 1996.

[18] B. Anna, Axicon imaging by scalar diffraction theory, Ph.D. thesis, Mikroelektronik och informationsteknik, Mikroelektronik och Informationsteknik, Stockholm, Sweden, 2004.

[19] M. Born and E. Wolf, Principles of Optics: Electromagnetic Theory of Propagation, Interference and Diffraction of Light, Cambridge University Press, Cambridge, UK, 7th edition, 1999.

[20] J. S. Jakob, "Waves, rays, and the method of stationary phase," Optics Express, vol. 10, pp. 740-751, 2002.

[21] J. Sochacki, A. Kołodziejczyk, Z. Jaroszewicz, and S. Bará, "Nonparaxial design of generalized axicons," Applied Optics, vol. 31, no. 25, pp. 5326-5330, 1992.

[22] A. A. Alkelly, M. Shukri, and Y. S. Alarify, "Influences of twist phenomenon of partially coherent field with uniform-intensity diffractive axicons," Journal of the Optical Society of America A, vol. 29, no. 4, pp. 417-425, 2012. 